\title{
Multiuser Rate Allocation Games for Multimedia Communications
}

\author{
Yan Chen, Student Member, IEEE, Beibei Wang, Student Member, IEEE, and K. J. Ray Liu, Fellow, IEEE
}

\begin{abstract}
How to efficiently and fairly allocate data rate among different users is a key problem in the field of multiuser multimedia communication. However, most of the existing optimization-based methods, such as minimizing the weighted sum of the distortions or maximizing the weighted sum of the peak signal-to-noise ratios (PSNRs), have their weights heuristically determined. Moreover, those approaches mainly focus on the efficiency issue while there is no notion of fairness. In this paper, we address this problem by proposing a game-theoretic framework, in which the utility/payoff function of each user/player is jointly determined by the characteristics of the transmitted video sequence and the allocated bit-rate. We show that a unique Nash equilibrium (NE), which is proportionally fair in terms of both utility and PSNR, can be obtained, according to which the controller can efficiently and fairly allocate the available network bandwidth to the users. Moreover, we propose a distributed cheat-proof rate allocation scheme for the users to converge to the optimal NE using alternative ascending clock auction. We also show that the traditional optimization-based approach that maximizes the weighted sum of the PSNRs is a special case of the game-theoretic framework with the utility function defined as an exponential function of PSNR. Finally, we show several experimental results on real video data to demonstrate the efficiency and effectiveness of the proposed method.
\end{abstract}

Index Terms-Cheat-proof, game theory, multimedia, Nash equilibrium, proportional fairness, rate allocation.

\section{INTRODUCTION}

$\mathbf{N}$ OWADAYS, due to the explosive growth of the Internet and the advance of compression technologies, delay-sensitive multimedia networking applications such as multimedia streaming and multicamera surveillance become more and more popular. Therefore, a fundamental problem in these applications, how to fairly and efficiently allocate the rate among many users who share the same network bandwidth, becomes more and more important and draws great attention recently.

Rate allocation for a single user has been well investigated in the literature [1]-[3]. In single-user rate allocation, the task of the rate controller is to assign the available rate to each frame and each macroblock (MB) to achieve the maximal visual quality. This is also known as rate control. The simplest rate control method is the constant bit-rate allocation (CBR),

Manuscript received September 30, 2008, revised May 27, 2009. Current version published September 16, 2009. The associate editor coordinating the review of this manuscript and approving it for publication was Prof. Aggelos K. Katsaggelos.

The authors are with the Department of Electrical and Computer Engineering, University of Maryland, College Park, MD 20742 USA (e-mail: yan@ umd.edu; bebewang@umd.edu; kjrliu@umd.edu).

Color versions of one or more of the figures in this paper are available online at http://ieeexplore.ieee.org.

Digital Object Identifier 10.1109/TMM.2009.2026101 which equally allocates the bit-rate to each frame. However, CBR often results in quality fluctuation, due to which the overall visual quality is significantly degraded. To overcome this problem, variable bit-rate allocation (VBR) is proposed for constant quality reconstruction by assigning rate according to the complexity of each frame [4]. A core technique in VBR-based rate control methods is rate distortion modeling [5], which highly affects the rate control performance. Many works have been done on rate distortion modeling, including parametric method [6] and nonparametric method [7].

If a channel is shared by multiple users, besides considering the rate allocation within the same user (i.e., frame-level rate allocation and MB-level rate allocation), the rate controller needs to consider the rate allocation among different users. This becomes the multiuser rate allocation problem. Similar to framelevel rate allocation, the simplest multiuser rate allocation is the CBR, where the available network bandwidth is equally assigned to each user. A major problem of CBR is that it does not consider the variable bit-rate characteristics of the video sequences. One way to overcome this disadvantage is to optimize a global objective function that involves the characteristics of all the video sequences using conventional optimization methods such as Lagrangian or dynamic programming [8]. For example, a commonly adopted method is for the rate controller to minimize the weighted sum of the distortions or try to maximize the weighted sum of the PSNRs, i.e., the optimization problem becomes

$$
\min _{R_{i}} \sum_{i=1}^{N} w_{i} D_{i}\left(R_{i}\right), \text { s.t. } \sum_{i=1}^{N} R_{i} \leq R
$$

or

$$
\max _{R_{i}} \sum_{i=1}^{N} w_{i} P S N R_{i}\left(R_{i}\right), \text { s.t. } \sum_{i=1}^{N} R_{i} \leq R
$$

where $R$ is the available network bandwidth, $w_{i}$ is the weight, $D_{i}$ is the distortion, and $P S N R_{i}$ is the PSNR of the $i$ th user.

Notice that the solution to the above optimization-based methods is highly related to the selection of the weights $w_{i}$. However, in the literature, the weights $w_{i}$ 's are usually heuristically determined, e.g., $w_{i}$ is uniformly set to be $1 / N$ [9]. Moreover, such a formulation can only address the efficiency issue, e.g., how to maximize the weighted sum of the PSNRs or minimize the weighted sum of the distortions. As such, the fairness issue, which is an important problem for multiuser rate allocation, has been generally ignored in the image/video/multimedia community.

However, in the networking literature, the fairness issue in multiuser rate allocation have been considered in a different 
setting. In [10], the authors formulated the optimal channelassignment problem as a convex optimization problem using a max-min fairness criterion for the downlink application. As pointed out in [11], the max-min approach deals with the worstcast scenario, so it favors users with worse channels and reduces the system efficiency. To overcome the disadvantage, the authors in [11] considered a generalized proportional fairness based on the Nash bargaining solutions and coalitions. While this proportional fairness criterion was successfully employed in networking applications, it cannot be directly used in contentaware multimedia applications since it does not explicitly consider the characteristics of the video content and the resulting impact on video quality. In [12], the authors applied the Nash bargaining solutions to the multimedia multiuser rate allocation problem, where the utility function for each user is defined as the inverse of the distortion. But there are two main drawbacks of that utility function. Firstly, since no cost in video transmission is considered, every user can overclaim his/her need to get more bandwidth regardless of the consequence to the system, which is recognized as selfish behavior. Due to the selfish nature, without a cost, all users will become too greedy and want to get as much bit-rate as possible, which is not good to the system [13]. Secondly, since the gain is defined as the inverse of the distortion, i.e., an exponential function of the peak signal-to-noise ratio (PSNR), a certain increase of the bit-rate in the low PSNR region will lead to a less significant gain than that in the high PSNR region. This contradicts with the human visual system (HVS) model since the quality difference in the low PSNR region is easier to be distinguished than that in the high PSNR region (see Section II-C for details). Moreover, with the utility function defined in [12], the generalized Nash bargaining solution is shown to be the same as the traditional optimization-based approach in (2), i.e., to maximize the weighted sum of the PSNRs, while the weights are determined by the bargaining powers, which are still heuristically determined.

In this paper, we propose a multiuser rate allocation game framework to efficiently and fairly allocate the available network bandwidth to different multimedia users. The utility/payoff function of each user/player is defined according to the characteristics of the transmitted video sequences and the allocated bit-rate. Specifically, motivated by the intuition that the quality difference in the low PSNR region is easier to be distinguished than that in the high PSNR region, we define the gain as a logarithm function of the PSNR. We also introduce a cost term in the utility function, which is linear in the allocated rate, to guide users' behaviors. In this way, the users will be more rational in choosing bit-rate since transmitting data with a higher bit-rate in this case does not necessarily result in a higher payoff, especially when the transmitted video sequence is a fast motion and complex scene sequence. Then, we discuss the Nash equilibrium (NE) of this rate allocation game. We show that with a unique NE, which is proportionally fair in terms of both utility and PSNR, can be obtained, based on which the rate controller can efficiently and fairly allocate the available rate. Moreover, we propose a decentralized cheat-proof rate allocation scheme for the users to converge to the unique $\mathrm{NE}$ using alternative ascending clock auction [14]. We also show that the traditional optimization-based method in (2) is a special

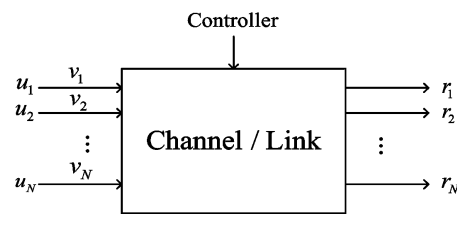

Fig. 1. System model.

case of the game-theoretic framework if the utility function is defined as an exponential function of PSNR. This fact indicates that the game-theoretic approach offers a more general and unified solution, especially in a multiuser setting. Finally, we illustrate several experimental results on real video data to demonstrate the efficiency and effectiveness of the proposed game-theoretic multiuser multimedia rate allocation method.

The rest of this paper is organized as follows. In Section II, we give a detailed description on the proposed method, including the system model, how to define the utility function, and the problem formulation. In Section III, we provide a detailed analysis of the proposed game-theoretic framework. In Section IV, we show the relationship between the proposed game-theoretic method and the traditional optimization-based approach. In Section $\mathrm{V}$, we describe in details the proposed distributed cheatproof rate allocation scheme using alternative ascending clock auction. Finally, we illustrate the experimental results on real video signals in Section VI and draw conclusions in Section VII.

\section{GAME-THEORETIC FRAMEWORK}

\section{A. System Model}

As shown in Fig. 1, in our system, we assume that there is a controller, $N$ transmitters, $u_{1}, u_{2}, \ldots, u_{N}$, and $N$ receivers, $r_{1}, r_{2}, \ldots, r_{N}$. User $u_{i}$ transmits the video sequence $v_{i}$ to the corresponding receiver $r_{i}$ through a channel/link that is shared by other users $u_{1}, \ldots, u_{i-1}, u_{i+1}, \ldots, u_{N}$. Since the channel has a limited bandwidth, it may not be able to satisfy the bandwidth requirements for all users. The role of the controller is to allocate the channel bandwidth to users $u_{1}, u_{2}, \ldots, u_{N}$. So, the question is how does the controller allocate the bandwidth to the users in an efficient and fair way? We will formally define the notion of fairness later.

\section{B. Video Distortion-Rate Model}

Before answering the question raised in the above subsection, let us first discuss the distortion-rate (DR) model for the video sequences. In video compression, due to the quantization process, there exists a tradeoff between the distortion $(D)$, which is usually defined as the mean squared error (MSE), and bit-rate $(R)$, which determines the channel bandwidth or storage space required to transmit or store the coded data. Generally, high bit-rate leads to small distortion while low bit-rate causes large distortion. In the literature, several models have been proposed to characterize this distortion rate tradeoff for different video coders, such as MPEG2 [3], [15], MPEG4 [1], [2], FGS [16], H.263 [17], H.264 [18], [19], and wavelet-based coders [20]. Without loss of generality, in this paper, we use a simple two-parameter distortion-rate model, which is widely employed in a medium or high bit-rate situation, and other models can be 
similarly analyzed. The two-parameter distortion-rate model is described as follows:

$$
D(R)=\alpha e^{-\beta R}
$$

where $\alpha$ and $\beta$ are two positive parameters determined by the characteristics of the video content.

\section{User's Utility Function}

As shown in Fig. 1, user $u_{i}$ can get gain by successfully transmitting the video $v_{i}$ to receiver $r_{i}$, and the gain is determined by the quality of the transmitted video. On the other hand, user $u_{i}$ needs to pay for the used bandwidth to transmit $v_{i}$, and the payment is determined by the bit-rate of $v_{i}$. Therefore, given the profile of $u_{i}$, the bit-rate $R_{i}$ and distortion $D_{i}$, the utility function of user $u_{i}$ can be defined as

$$
U_{i}\left(R_{i}, D_{i}\right)=f\left(D_{i}\right)-a g\left(R_{i}\right)
$$

where $f\left(D_{i}\right)$ is the gain, $g\left(R_{i}\right)$ is the cost, and $a$ is a parameter controlling the balance between the gain and cost.

Generally, since the gain of $u_{i}$ will be larger if the distortion $D_{i}$ is smaller, the function $f(\cdot)$ should be a monotonically decreasing function. Similarly, since the cost of $u_{i}$ will be larger if the bit-rate $R_{i}$ is larger, the function $g(\cdot)$ should be a monotonically increasing function. Without loss of generality, we assume that the cost per bit-rate unit is one, which means

$$
g\left(R_{i}\right)=R_{i}
$$

The gain $f\left(D_{i}\right)$ is generally determined by how much receiver $r_{i}$ is satisfied with the received video. In video processing and coding community, the PSNR is a more common objective quality measure than MSE. For any MSE, i.e., the distortion $D$, the corresponding PSNR is given by

$$
P S N R=10 \log _{10} \frac{255^{2}}{D} .
$$

Moreover, according to the human visual system (HVS) model, the quality difference in the low PSNR region is easier to be distinguished than that in the high PSNR region, e.g., as shown in Fig. 2, the $33 \mathrm{~dB}$ and $34 \mathrm{~dB}$ images are easier to be distinguished than the $40 \mathrm{~dB}$ and $41 \mathrm{~dB}$ images. Therefore, we define the $f(\cdot)$ function as

$$
f\left(D_{i}\right)=\ln \left(P S N R_{i}\right)=\ln \left[10 \log _{10} \frac{255^{2}}{D_{i}}\right] .
$$

Note that the reason of using $\ln (\cdot)$ function is that $\ln (\cdot)$ is a monotonically increasing function in its argument and its second order derivative is negative, due to which a certain increase in the low PSNR region will lead to a more significant gain than that in the high PSNR region. Other functions that have similar properties can also be used. Moreover, if we do not consider the distinct characteristics of video signals, any monotonically decreasing function of the distortion $D$ can be used, e.g.,

$$
f(D)=\frac{255^{2}}{D}=e^{\eta P S N R}
$$

or

$$
f(D)=10 \log _{10} \frac{255^{2}}{D}=P S N R .
$$

Combining (3)-(7) and ignoring the constant term, the utility function of user $u_{i}$ becomes

$$
U_{i}\left(R_{i}\right)=\ln \left(\gamma_{i}+\beta_{i} R_{i}\right)-a R_{i}
$$

where $\gamma_{i}=2 \ln 255-\ln \alpha_{i}$.

\section{Multiuser Rate Allocation Game}

To answer the question raised in Section II-A, we formulate this problem as a multiuser rate allocation game. As shown in Fig. 1, in this game, there are $N$ users/players, who share the available network bandwidth with each other. Each user $u_{i}$ has his/her own utility function as shown in (10), and it also has a minimum desired quality constraint (minimal rate constraint $R_{i}^{\mathrm{min}}$ ) and a maximum satisfied quality constraint (maximum rate constraint $R_{i}^{\max }$ ). Since $R_{i}^{\min }$ is the minimal rate constraint that each user expects by jointing in the game, we assume that the available network rate at least guarantees each user for the minimal desired rate in the game. Obviously, if the available network bandwidth is able to satisfy all the users with the maximum quality constraint $R_{i}^{\max }$, the rate allocation problem is trivial since the controller just needs to allocate $R_{i}^{\max }$ to each user $u_{i}$. However, in the case that the available network bandwidth is not able to satisfy all the users with $R_{i}^{\max }$, the problem becomes more interesting: how does the controller fairly and efficiently allocate the available bandwidth to the users? From the users' point of view, they try to maximize their utilities subject to the constraint that the sum of the users' bit-rate does not exceed the available bandwidth. Therefore, the game can be formulated as

$$
\begin{array}{ll}
\max _{R_{i}} & U_{i}\left(R_{i}\right)=\ln \left(\gamma_{i}+\beta_{i} R_{i}\right)-a R_{i} \\
\text { s.t. } & R_{i}^{\min } \leq R_{i} \leq R_{i}^{\max }, \quad \forall i=1,2, \ldots, N \\
& \sum_{i=1}^{N} R_{i} \leq R
\end{array}
$$

where $R$ is the available network bandwidth.

\section{Analysis of the Multiuser Rate Allocation Game}

According to (10), we can see that the utility function $U_{i}\left(R_{i}\right)$ is a concave function in terms of $R_{i}$. By taking the derivative of $U_{i}\left(R_{i}\right)$ over $R_{i}$, we have

$$
\frac{\partial U_{i}\left(R_{i}\right)}{\partial R_{i}}=\frac{\beta_{i}}{\gamma_{i}+\beta_{i} R_{i}}-a, \quad \forall i=1,2, \ldots, N .
$$

Therefore, user $u_{i}$ achieves his/her maximal utility $U_{i}^{\star}\left(R_{i}^{\star}\right)$ at $R_{i}^{\star}$, where $R_{i}^{\star}$ is defined as

$$
\begin{aligned}
& R_{i}^{\star}=\max \left[R_{i}^{\min }, \min \left(\frac{1}{a}-\frac{\gamma_{i}}{\beta_{i}}, R_{i}^{\max }\right)\right] \\
& \forall i=1,2, \ldots, N .
\end{aligned}
$$




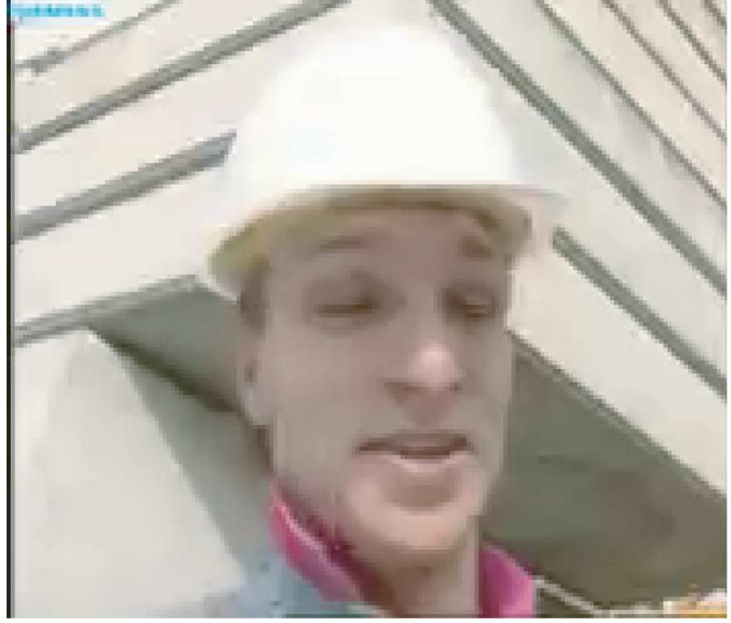

(a)

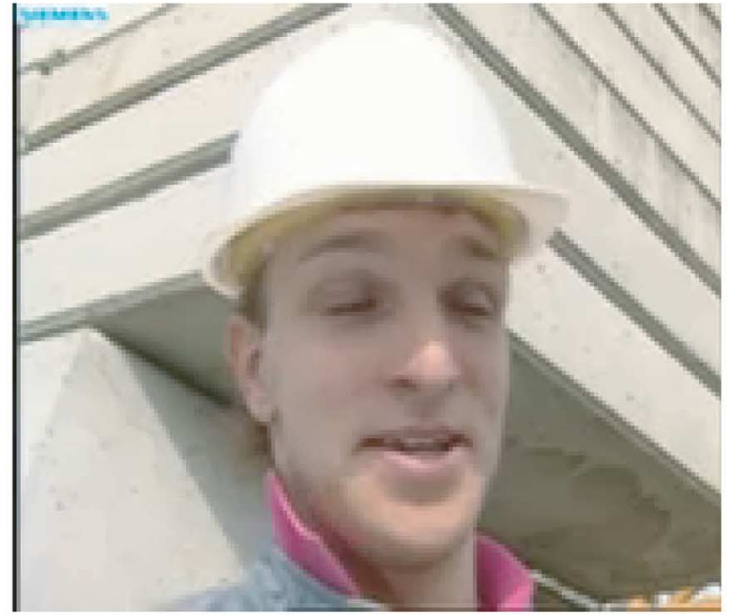

(c)

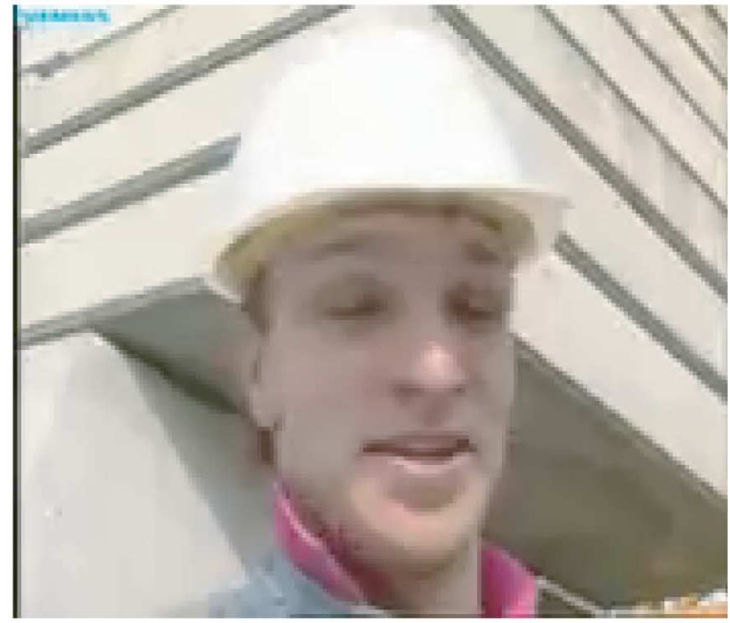

(b)

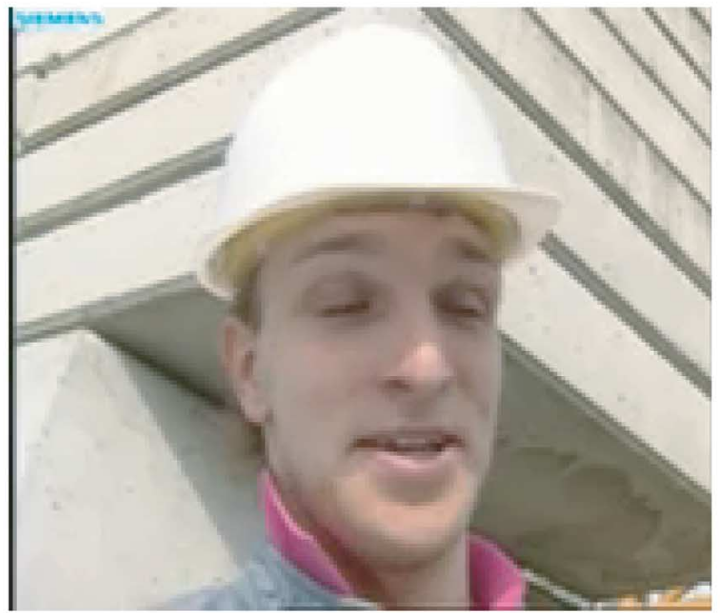

(d)

Fig. 2. Visual quality of Foreman sequence at different PSNR level. (a) 33 dB. (b) 34 dB. (c) 40 dB. (d) 41 dB.

From (13), we can see that the optimal $R_{i}^{\star}$ corresponding to the maximal utility is determined by the parameter $a$. Therefore, for different choices of $a$, the game in (11) has different equilibria with different physical meanings. Specifically, in the following, we discuss the Nash equilibrium (NE) in three different cases: $a>a_{0}, 0 \leq a<a_{0}$, and $a=a_{0}$, where $a_{0}$ is the constant that satisfies the following equation:

$$
\sum_{i=1}^{N}\left(\max \left[R_{i}^{\min }, \min \left(\frac{1}{a_{0}}-\frac{\gamma_{i}}{\beta_{i}}, R_{i}^{\max }\right)\right]\right)=R
$$

\section{A. Non-Efficient Rate Allocation $\left(a>a_{0}\right)$}

If $a>a_{0}$, the game in (11) has a unique Nash equilibrium $\left(R_{1}^{\star}, R_{2}^{\star}, \ldots, R_{N}^{\star}\right)$. Since $a>a_{0}$, from (14), we have $\sum_{i=1}^{N} R_{i}^{\star}<R$, which means that the available network bandwidth is not fully utilized. Therefore, this allocation scheme is not efficient.

\section{B. Efficient Rate Allocation $\left(0 \leq a<a_{0}\right)$}

If $0 \leq a<a_{0}$, the game in (11) has infinitely many NE. For every $\operatorname{NE}\left(\tilde{R}_{1}, \tilde{R}_{2}, \ldots, \tilde{R}_{N}\right)$, according to Lemma 1 , we have $\sum_{i=1}^{N} \tilde{R}_{i}=R$, which means that the available network bandwidth is fully utilized. Therefore, this allocation scheme is efficient.

Lemma 1: When $0 \leq a<a_{0}$, every NE $\left(\tilde{R}_{1}, \tilde{R}_{2}, \ldots, \tilde{R}_{N}\right)$ satisfies $\sum_{i=1}^{N} \tilde{R}_{i}=R$.

Proof: Since $\sum_{i=1}^{N} \tilde{R}_{i} \leq R$, let us assume that there is an NE $\left(\tilde{R}_{1}, \tilde{R}_{2}, \ldots, \tilde{R}_{N}\right)$ such that $\sum_{i=1}^{N} \tilde{R}_{i}=R-\Delta<R$. Since $0 \leq a<a_{0}$, we have $\sum_{i=1}^{N} R_{i}^{\star}>R$, which means there exists at least one $\tilde{R}_{j}$ such that $\tilde{R}_{j}<R_{j}^{\star}$. Let $\hat{R}_{j}=$ $\min \left(\tilde{R}_{j}+\Delta, R_{j}^{\star}\right)$, then $\sum_{i=1}^{j-1} \tilde{R}_{i}+\hat{R}_{j}+\sum_{i=j+1}^{N} \tilde{R}_{i} \leq R$ and $U_{j}\left(\hat{R}_{j}\right)>U_{j}\left(\tilde{R}_{j}\right)$ (due to the concavity of the utility function). This contradicts with the assumption that $\left(\tilde{R}_{1}, \tilde{R}_{2}, \ldots, \tilde{R}_{N}\right)$ is an NE. Therefore, $\sum_{i=1}^{N} \tilde{R}_{i}=R$. This completes the proof.

\section{Efficient and Proportionally Fair in Both Utility and PSNR $\left(a=a_{0}\right)$}

If $a=a_{0}$, the game in (11) has a unique Nash equilibrium $\left(R_{1}^{\star}, R_{2}^{\star}, \ldots, R_{N}^{\star}\right)$. According to (14), we have $\sum_{i=1}^{N} R_{i}^{\star}=R$, which means that the available network bandwidth is fully utilized. Therefore, this allocation scheme is efficient. 
Moreover, we will show in the following definition [21] and theorem that when $a=a_{0},\left(R_{1}^{\star}, R_{2}^{\star}, \ldots, R_{N}^{\star}\right)$ is a proportionally fair NE in terms of both utility and PSNR.

Definition 1: A utility distribution is said to be proportionally fair when any change in the distribution of utilities results in the sum of the proportional changes being non-positive, i.e.,

$$
\sum_{i} \frac{U_{i}-\tilde{U}_{i}}{\tilde{U}_{i}} \leq 0, \quad \forall U_{i} \in \mathbf{S}
$$

where $\tilde{U}_{i}$ and $U_{i}$ are the proportionally fair utility and any other feasible utility for the $i$ th user, respectively, and $\mathbf{S}$ is a closed and convex subset of $\Re^{N}$ to represent the set of feasible utility functions that the users can achieve.

Remark: The definition of proportional fairness comes from the fact that, if $\left(\tilde{U}_{1}, \tilde{U}_{2}, \ldots, \tilde{U}_{N}\right)$ satisfied (15), any deviation from $\left(\tilde{U}_{1}, \tilde{U}_{2}, \ldots, \tilde{U}_{N}\right)$ will lead to a non-increasing sum of the proportional changes. Moreover, from [21] and [11], we can see that $\left(\tilde{U}_{1}, \tilde{U}_{2}, \ldots, \tilde{U}_{N}\right)$ is a proportionally fair utility if and only if $\prod_{i=1}^{N} \tilde{U}_{i} \geq \prod_{i=1}^{N} U_{i}$ for any feasible $\left(U_{1}, U_{2}, \ldots, U_{N}\right)$.

Theorem 1: When $a=a_{0},\left(R_{1}^{\star}, R_{2}^{\star}, \ldots, R_{N}^{\star}\right)$ is a proportionally fair NE in terms of both utility and PSNR.

Proof: According to the above remark, $\left(R_{1}^{\star}, R_{2}^{\star}, \ldots, R_{N}^{\star}\right)$ is a proportionally fair NE in terms of both utility and PSNR if and only if it is the solution to the following two optimization problems:

$$
\begin{array}{ll}
\max _{R_{i}} & \prod_{i=1}^{N} U_{i}=\prod_{i=1}^{N}\left[\ln \left(\gamma_{i}+\beta_{i} R_{i}\right)-a R_{i}\right] \\
\text { s.t. } & R_{i}^{\min } \leq R_{i} \leq R_{i}^{\max }, \quad \forall i=1,2, \ldots, N \\
& \sum_{i=1}^{N} R_{i} \leq R
\end{array}
$$

and

$$
\begin{array}{ll}
\max _{R_{i}} & \prod_{i=1}^{N} P S N R_{i}=\prod_{i=1}^{N}\left(\gamma_{i}+\beta_{i} R_{i}\right) \\
\text { s.t. } & R_{i}^{\min } \leq R_{i} \leq R_{i}^{\max }, \quad \forall i=1,2, \ldots, N \\
& \sum_{i=1}^{N} R_{i} \leq R .
\end{array}
$$

- We first show that $\left(R_{1}^{\star}, R_{2}^{\star}, \ldots, R_{N}^{\star}\right)$ is a proportionally fair NE in terms of utility. Since user $u_{i}$ achieves his/her maximal utility $U_{i}^{\star}\left(R_{i}^{\star}\right)$ at $R_{i}^{\star}$, we have $U_{i}\left(R_{i}^{\star}\right) \geq U_{i}\left(R_{i}\right)$, for any $R_{i}$ satisfies $R_{i}^{\min } \leq R_{i} \leq R_{i}^{\max }$ and $\sum_{i=1}^{N} R_{i} \leq R$. This means that $\prod_{i=1}^{N} U_{i}\left(R_{i}^{\star}\right) \geq \prod_{i=1}^{N} U_{i}\left(R_{i}\right)$ for any feasible $\left(R_{1}, R_{2}, \ldots, R_{N}\right)$. Therefore, $\left(R_{1}^{\star}, R_{2}^{\star}, \ldots, R_{N}^{\star}\right)$ is a proportionally fair NE in terms of utility.

- We then show that $\left(R_{1}^{\star}, R_{2}^{\star}, \ldots, R_{N}^{\star}\right)$ is a proportionally fair NE in terms of PSNR. Since maximizing $\prod_{i} x_{i}$ is the same as maximizing $\sum_{i} \ln \left(x_{i}\right)$, the optimization problem in (17) is equivalent to the following optimization problem:

$$
\begin{array}{ll}
\max _{R_{i}} & \sum_{i=1}^{N} \ln \left(\gamma_{i}+\beta_{i} R_{i}\right) \\
\text { s.t. } & R_{i}^{\min } \leq R_{i} \leq R_{i}^{\max }, \quad \forall i=1,2, \ldots, N \\
& \sum_{i=1}^{N} R_{i} \leq R .
\end{array}
$$

Since the above optimization problem is convex, the optimal solution can be found by solving the Karush-Kuhn-Tucker (KKT) conditions [22]. We first write the Lagrangian of problem (18) as

$$
\begin{aligned}
L\left(R_{i}, \lambda, \kappa_{i}, \nu_{i}\right) & \\
= & -\sum_{i=1}^{N} \ln \left(\gamma_{i}+\beta_{i} R_{i}\right)+\lambda\left(\sum_{i=1}^{N} R_{i}-R\right) \\
& +\sum_{i=1}^{N} \kappa_{i}\left(R_{i}-R_{i}^{\max }\right)+\sum_{i=1}^{N} \nu_{i}\left(R_{i}^{\min }-R_{i}\right) .
\end{aligned}
$$

Then, the KKT conditions are

$$
\begin{aligned}
-\frac{\beta_{i}}{\gamma_{i}+\beta_{i} R_{i}}+\lambda+\kappa_{i}-\nu_{i} & =0 \\
\lambda\left(\sum_{i=1}^{N} R_{i}-R\right) & =0 \\
\kappa_{i}\left(R_{i}-R_{i}^{\max }\right) & =0, \quad \forall i=1, \ldots, N \\
\nu_{i}\left(R_{i}^{\min }-R_{i}\right) & =0, \quad \forall i=1, \ldots, N \\
R_{i}^{\min } \leq R_{i} & \leq R_{i}^{\max }, \quad \forall i=1, \ldots, N \\
\sum_{i=1}^{N} R_{i} & \leq R \\
\lambda & \geq 0, \quad \kappa_{i} \geq 0, \quad \nu_{i} \geq 0 \\
\forall i & =1, \ldots, N .
\end{aligned}
$$

By solving the KKT conditions above, the optimal solution is

$$
\begin{aligned}
& R_{i}^{\star}=\max \left[R_{i}^{\min }, \min \left(\frac{1}{\lambda}-\frac{\gamma_{i}}{\beta_{i}}, R_{i}^{\max }\right)\right] \\
& \forall i=1, \ldots, N
\end{aligned}
$$

where

$\sum_{i=1}^{N} \max \left[R_{i}^{\min }, \min \left((1 / \lambda)-\left(\gamma_{i} / \beta_{i}\right), R_{i}^{\max }\right)\right]=R$.

Therefore, $\left(R_{1}^{\star}, R_{2}^{\star}, \ldots, R_{N}^{\star}\right)$ is the solution to the optimization problem in (17), which means that it is a proportionally fair NE in terms of PSNR.

In all, when $a=a_{0},\left(R_{1}^{\star}, R_{2}^{\star}, \ldots, R_{N}^{\star}\right)$ is a proportionally fair NE in terms of both utility and PSNR. This completes the proof.

Remark: From the above analysis, we can see that choosing $\left(a=a_{0}\right)$ is the best among the three different cases due to the following four reasons: 1) a unique proportionally fair NE in terms of both utility and PSNR can be found when $a=a_{0} ; 2$ ) with the unique proportionally fair NE, the available network bandwidth will be fully utilized; 3 ) since the optimal solution shown in (21) is very simple, no optimization is needed and the computational complexity is low; and 4) a distributed algorithm can be designed for the users to converge to the unique NE which will be discussed in Section V. 


\section{Relation to the TRADitional OPTIMIZATION-BASED APPROACH}

While the task of rate allocation for a single user is to find the best trade-off point on the rate-distortion curve, the traditional optimization-based multiuser rate allocation approach can be seen as first constructing an overall rate-distortion curve by combining rate-distortion curves of all users, and then finding the best trade-off point on the joint rate-distortion curve. However, it is difficult to construct the overall rate-distortion curve from all users' rate-distortion curve. The approach shown in (2) is one possible way, but there is no notion of fairness. Furthermore, the weights in (2) are hard to determine and are usually defined heuristically.

Instead of focusing on finding a good way of constructing the overall rate-distortion curve, the proposed game-theoretic framework considers each user's rate-distortion trade-off in the utility function. Then, the notion of proportional fairness is introduced to balance the rate allocation among different users and to make sure that the total rate constraint is satisfied. Moreover, from (2), (4), and (16), we can see that the traditional optimization-based approach shown in (2) is actually a special case of the proposed game-theoretic framework by choosing the gain function and the cost function as follows:

$$
f\left(D_{i}\right)=e^{w_{i} P S N R_{i}}, \quad g\left(R_{i}\right)=0
$$

which means

$$
U_{i}\left(R_{i}, D_{i}\right)=e^{w_{i} P S N R_{i}}
$$

Note that there are mainly three drawbacks of this kind of utility function.

- The parameters $w_{i}$ are usually heuristically determined.

- If no cost in video transmission is considered, selfish users may become too greedy and want to get as much bit-rate as possible, which is not good to the system [13].

- Since the gain is defined as an exponential function of the PSNR, a certain increase of the bit-rate in the low PSNR region will lead to a less significant gain than that in the high PSNR region. This contradicts with the HVS model since the quality difference in the low PSNR region is easier to be distinguished than that in the high PSNR region.

\section{Clock Auction for Distributed Cheat-Proof OPTIMAL RATE ALLOCATION}

In Section III, we have discussed the NE of the multiuser rate allocation game for different $a$ 's and found that, when $a=a_{0}$, the game has a proportionally fair NE in both utility and PSNR. However, we have not discussed how to obtain $a_{0}$ and how the users converge to the NE yet. There are two possible approaches: centralized approach and distributed approach. For the centralized approach, the controller knows exactly all the private information of each user, i.e., $\gamma_{i}, \beta_{i}, R_{i}^{\min }$, and $R_{i}^{\max }$. Then, the controller can first find $a_{0}$ in a collective way by solving (14) and then allocate $R_{i}^{\star}$ to $u_{i}$.

However, in general, the users can be geographically distributed in many places; it is therefore not feasible for the controller to collect all the private information of each user. Moreover, since the users are selfish, e.g., they tend to overclaim what they may need, they will not truly report their private information if cheating can improve their utilities [23]. To solve this problem, we propose a distributed cheat-proof rate allocation scheme using alternative ascending clock auction [14]. An auction is a decentralized mechanism for allocating resources, where there is an auctioneer and several bidders. The auction processes can be described as follows: the auctioneer announces a price, bidders report to the auctioneer their demands at that price, and the auctioneer raises the price until the total demand meets the supply. In our multiuser rate allocation problem, the controller is the auctioneer and the users are the bidders.

The proposed rate allocation scheme is described in Algorithm 1. As shown in Algorithm 1, before the auction, the controller sets up the step size $\delta>0$, clock index $t=0$, and initializes $a$ with a small value $a^{0}$. At the beginning of clock $t$, the controller first announces $a^{t}$ to all the users. Then, each user submits his/her optimal demand to the controller. After collecting all the demands, the controller compares the total demand $R_{\text {total }}$ with the available bandwidth $R$. If $R_{\text {total }}>R$, i.e., the total demand exceeds the supply, the auction is not concluded. The controller continues the auction and goes to next clock $t+1$ with an increased $a$ computed by $a^{t+1}=a^{t}+\delta$. Moreover, the controller computes the cumulative clinch, which is the amount of bit-rate that the user is guaranteed to win at current clock, for each user given by

$$
C_{i}^{t}=\max \left(0, R-\sum_{j \neq i} R_{j}^{t}\right) .
$$

Algorithm 1: Cheat-Proof Rate Allocation Scheme Using Clock Auction

Given the available bandwidth $R$, step size $\delta>0$, and clock index $t=0$, the controller initializes $a$ with a small value $a^{0}$.

Repeat:

1) the controller announces $a^{t}$ to all the users.

2) Each user $u_{i}$ submits his/her optimal demand:

$$
R_{i}^{t}=\max \left[R_{i}^{\min }, \min \left(\frac{1}{a^{t}}-\frac{\gamma_{i}}{\beta_{i}}, R_{i}^{\max }\right)\right] .
$$

3) The controller sums up all the demand $R_{\text {total }}^{t}=\sum_{i} R_{i}^{t}$ and compares $R_{\text {total }}^{t}$ with $R$ :

If $R_{\text {total }}^{t}>R$, compute $C_{i}^{t}=\max \left(0, R-\sum_{j \neq i} R_{j}^{t}\right)$, set $a^{t+1}=a^{t}+\delta, t=t+1$, and go to 1$)$.

Else, conclude the auction, set $L=t$, compute

$$
C_{i}^{L}=R_{i}^{L}+\frac{R_{i}^{L-1}-R_{i}^{L}}{\sum_{i} R_{i}^{L-1}-\sum_{i} R_{i}^{L}}\left[R-\sum_{i} R_{i}^{L}\right]
$$

and allocate $R_{i}^{\star}=C_{i}^{L}$ to $u_{i}$.

Finally, the payment of $u_{i}$ is $P_{i}^{\star}=C_{i}^{0} a^{0}+$ $\sum_{t=1}^{L} a^{t}\left(C_{i}^{t}-C_{i}^{t-1}\right)$ and the utility of $u_{i}$ is $U_{i}^{\star}=\ln \left(\gamma_{i}+\beta_{i} R_{i}^{\star}\right)-P_{i}^{\star}$. 
On the other hand, if $R_{\text {total }} \leq R$, then the supply can meet all users' demands and the auction is concluded. Let the final clock index be $L$. As $a$ increases discretely, we may have $R_{\text {total }}<$ $R$ and do not fully utilize the bandwidth. To make sure that $R_{\text {total }}=R$, we modify (24) by introducing proportional rationing [14]. Then, the final cumulative clinch of $u_{i}$ is given by

$$
C_{i}^{L}=R_{i}^{L}+\frac{R_{i}^{L-1}-R_{i}^{L}}{\sum_{i} R_{i}^{L-1}-\sum_{i} R_{i}^{L}}\left[R-\sum_{i} R_{i}^{L}\right]
$$

$$
\text { with } \sum_{i} C_{i}^{L}=R \text {. }
$$

Finally, the rate allocated to $u_{i}$ is $R_{i}^{\star}=C_{i}^{L}$. The utility of $u_{i}$ is obtained as

$$
U_{i}^{\star}=\ln \left(\gamma_{i}+\beta_{i} R_{i}^{\star}\right)-P_{i}^{\star}
$$

where $P_{i}^{\star}=C_{i}^{0} a^{0}+\sum_{t=1}^{L} a^{t}\left(C_{i}^{t}-C_{i}^{t-1}\right)$ is the payment from user $u_{i}$.

Remark: Since $a^{t+1}>a^{t}$, we have $R_{j}^{t+1} \leq R_{j}^{t}$. Therefore, at clock $t, u_{i}$ is guaranteed at least the amount of bit-rate $C_{i}^{t}=$ $\max \left(0, R-\sum_{j \neq i} R_{j}^{t}\right)$. This is where(24) comes from.

The rate allocation scheme described in Algorithm 1 has several advantages.

- The auction process is transparent to all users and simple enough for all users to understand. Simplicity and transparency are two important factors to stimulate auction since users may not be willing to join in the game if they do not understand the auction process.

- The auction scheme can preserve privacy. Since the scheme is distributed, users do not need to report their private information. Instead, they only need to submit their demands.

- The computational complexity of each user is low since what the users need to do is to submit their optimal demands calculated by $R_{i}=\max \left[R_{i}^{\min }, \min \left((1 / a)-\left(\gamma_{i} / \beta_{i}\right), R_{i}^{\max }\right)\right]$ for any given $a$.

- The computational complexity of the controller is low in that the controller only needs to sum up the demands from the users, compare it with the available bandwidth, and compute the cumulative clinch for each user.

- Through the auction, each user will converge to the unique proportionally fair NE shown in Section III. This is trivial due to the following two reasons: 1) since the auction concludes if and only if $\sum_{i} R_{i}^{t} \leq R$, when $\delta$ is sufficient small, the auction will conclude at $\sum_{i} R_{i}^{t}=R$. 2) At each clock $t, u_{i}$ chooses $R_{i}^{t}=\max \left[R_{i}^{\min }, \min \left(\left(1 / a^{t}\right)-\left(\gamma_{i} / \beta_{i}\right), R_{i}^{\max }\right)\right]$.

- The scheme is cheat-proof, meaning that the best strategy of each user is to report his/her true optimal demand at every clock. There is no incentive for $u_{i}$ to deviate, and the proof is shown in Theorem 2.

Let $R_{i}^{t}$ be user $u_{i}$ 's true optimal demand at clock $t$, and $\tilde{R}_{i}{ }^{t}$ be the claimed demand that $u_{i}$ reports to the controller at clock $t$. Note that $\tilde{R}_{i}^{t}$ can be any value in $\left[R_{i}^{\min }, R_{i}^{\max }\right]$ if $u_{i}$ cheats at clock $t$. Let $\Gamma(t, L)=$
$\left\{L, \tilde{R}_{i}{ }^{0}, \ldots, \tilde{R}_{i}{ }^{t}, R_{i}^{t+1}, \ldots, R_{i}^{L} ; C_{i}^{0}, \ldots, C_{i}^{L} ; a^{0}, \ldots, a^{L}\right\}$

be the profile of $u_{i}$ at the following scenario: from clock 0 to clock $t, u_{i}$ reports $\tilde{R}_{i}{ }^{0}, \ldots, \tilde{R}_{i}{ }^{t}$, and from clock $t+1$ to the final clock, $u_{i}$ reports $R_{i}^{t+1}, \ldots, R_{i}^{L}$, where $L$ is the final clock index, $C_{i}^{0}, \ldots, C_{i}^{L}$ is the corresponding cumulative clinch of $u_{i}$ from clock 0 to clock $L$, and $a^{0}, \ldots, a^{L}$ is the corresponding value of $a$ at each clock. Let $U_{i}[\Gamma(t, L)]$ be the utility of $u_{i}$ in this scenario. Let $\Gamma(-1, L)=\left\{L, R_{i}^{0}, \ldots, R_{i}^{L} ; C_{i}^{0}, \ldots, C_{i}^{L} ; a^{0}, \ldots, a^{L}\right\}$ and $\Gamma(L, L)=\left\{L, \tilde{R}_{i}{ }^{0}, \ldots, \tilde{R}_{i}{ }^{L} ; C_{i}^{0}, \ldots, C_{i}^{L} ; a^{0}, \ldots, a^{L}\right\}$ be two special cases of $\Gamma(t, L)$.

Lemma 2: If all other users report their true optimal demands at every clock, then $U_{i}\left[\Gamma\left(t-1, L_{1}\right)\right] \geq U_{i}\left[\Gamma\left(t, L_{2}\right)\right]$.

Proof: From (26), we have

$$
\begin{aligned}
U_{i}\left[\Gamma\left(t-1, L_{1}\right)\right]= & \ln \left(\gamma_{i}+\beta_{i} R_{i}^{L_{1}}\right)-a^{0} C_{i}^{0} \\
& -\sum_{k=1}^{L_{1}} a^{k}\left(C_{i}^{k}-C_{i}^{k-1}\right) \\
U_{i}\left[\Gamma\left(t, L_{2}\right)\right]= & \ln \left(\gamma_{i}+\beta_{i} R_{i}^{L_{2}}\right)-a^{0} C_{i}^{0} \\
& -\sum_{k=1}^{L_{2}} a^{k}\left(C_{i}^{k}-C_{i}^{k-1}\right) .
\end{aligned}
$$

- If $\tilde{R}_{i}^{t} \leq R_{i}^{t}$, according to Algorithm 1, we have $L_{2} \leq L_{1}$ and $R_{i}^{\overline{L_{2}}} \leq R_{i}^{L_{1}}$. Then

$$
\begin{aligned}
& U_{i}\left[\Gamma\left(t-1, L_{1}\right)\right]-U_{i}\left[\Gamma\left(t, L_{2}\right)\right] \\
& =\ln \left(\gamma_{i}+\beta_{i} R_{i}^{L_{1}}\right)-\ln \left(\gamma_{i}+\beta_{i} R_{i}^{L_{2}}\right) \\
& \quad-\sum_{k=L_{2}+1}^{L_{1}} a^{k}\left(C_{i}^{k}-C_{i}^{k-1}\right) \\
& \geq \ln \left(\gamma_{i}+\beta_{i} R_{i}^{L_{1}}\right)-\ln \left(\gamma_{i}+\beta_{i} R_{i}^{L_{2}}\right) \\
& \quad-a^{L_{1}}\left(C_{i}^{L_{1}}-C_{i}^{L_{2}}\right) .
\end{aligned}
$$

When $\delta$ is sufficiently small, $C_{i}^{L_{1}}=R_{i}^{L_{1}}$ and $C_{i}^{L_{2}}=R_{i}^{L_{2}}$ Since $R_{i}^{t}=\max \left[R_{i}^{\min }, \min \left(\left(1 / a^{t}\right)-\left(\gamma_{i} / \beta_{i}\right), R_{i}^{\max }\right)\right]$ —if $R_{i}^{L_{1}}>R_{i}^{\min }$, according to (12), we get $a^{L_{1}} \leq$ $\left(\partial \ln \left(\gamma_{i}+\beta_{i} R_{i}\right) /\left.\partial R_{i}\right|_{R_{i}=R_{i}^{L_{1}}}\right)$. Thus, (28) becomes

$$
\begin{aligned}
& U_{i}\left[\Gamma\left(t-1, L_{1}\right)\right]-U_{i}\left[\Gamma\left(t, L_{2}\right)\right] \\
& \geq\left[\ln \left(\gamma_{i}+\beta_{i} R_{i}^{L_{1}}\right)\right. \\
& \left.-\left.\frac{\partial \ln \left(\gamma_{i}+\beta_{i} R_{i}\right)}{\partial R_{i}}\right|_{R_{i}=R_{i}^{L_{1}}}\left(R_{i}^{L_{1}}-R_{i}^{L_{2}}\right)\right] \\
& -\ln \left(\gamma_{i}+\beta_{i} R_{i}^{L_{2}}\right) \\
& \geq 0 \text { (see Figure } 3(a) \text { ). }
\end{aligned}
$$

—if $R_{i}^{L_{1}}=R_{i}^{\min }$, since $R_{i}^{\min } \leq R_{i}^{L_{2}} \leq R_{i}^{L_{1}}=R_{i}^{\min }$, we have $R_{i}^{L_{2}}=R_{i}^{\min }=R_{i}^{L_{1}}$. Therefore

$$
U_{i}\left[\Gamma\left(t-1, L_{1}\right)\right]=U_{i}\left[\Gamma\left(t, L_{2}\right)\right] .
$$

So, if $\tilde{R}_{i}^{t} \leq R_{i}^{t}$, we have $U_{i}\left[\Gamma\left(t-1, L_{1}\right)\right] \geq U_{i}\left[\Gamma\left(t, L_{2}\right)\right]$. 
- If $\tilde{R}_{i}{ }^{t}>R_{i}^{t}$, according to Algorithm 1 , we have $L_{2} \geq L_{1}$ and $R_{i}^{L_{2}} \geq R_{i}^{L_{1}}$. Then

$$
\begin{aligned}
U_{i}\left[\Gamma\left(t-1, L_{1}\right)\right]-U_{i}\left[\Gamma\left(t, L_{2}\right)\right] \\
=\ln \left(\gamma_{i}+\beta_{i} R_{i}^{L_{1}}\right)-\ln \left(\gamma_{i}+\beta_{i} R_{i}^{L_{2}}\right) \\
\quad+\sum_{k=L_{1}+1}^{L_{2}} a^{k}\left(C_{i}^{k}-C_{i}^{k-1}\right) \\
\geq \ln \left(\gamma_{i}+\beta_{i} R_{i}^{L_{1}}\right)-\ln \left(\gamma_{i}+\beta_{i} R_{i}^{L_{2}}\right) \\
\quad+a^{L_{1}}\left(C_{i}^{L_{2}}-C_{i}^{L_{1}}\right) .
\end{aligned}
$$

When $\delta$ is sufficiently small, $C_{i}^{L_{1}}=R_{i}^{L_{1}}$ and $C_{i}^{L_{2}}=R_{i}^{L_{2}}$. Since $R_{i}^{t}=\max \left[R_{i}^{\min }, \min \left(\left(1 / a^{t}\right)-\left(\gamma_{i} / \beta_{i}\right), R_{i}^{\max }\right)\right]$ -if $R_{i}^{L_{1}}<R_{i}^{\max }$, according to (12), $a^{L_{1}} \geq$ $\left(\partial \ln \left(\gamma_{i}+\beta_{i} R_{i}\right) /\left.\partial R_{i}\right|_{R_{i}=R_{i}^{L_{1}}}\right)$. Thus, (31) becomes

$$
\begin{aligned}
& U_{i}\left[\Gamma\left(t-1, L_{1}\right)\right]-U_{i}\left[\Gamma\left(t, L_{2}\right)\right] \\
& \begin{array}{l}
\geq \ln \left(\gamma_{i}+\beta_{i} R_{i}^{L_{1}}\right) \\
\quad-\left[\ln \left(\gamma_{i}+\beta_{i} R_{i}^{L_{2}}\right)\right. \\
\left.\quad-\left.\frac{\partial \ln \left(\gamma_{i}+\beta_{i} R_{i}\right)}{\partial R_{i}}\right|_{R_{i}=R_{i}^{L_{1}}}\left(R_{i}^{L_{2}}-R_{i}^{L_{1}}\right)\right]
\end{array}
\end{aligned}
$$

$\geq 0$ [see Fig. 3(b)].

—if $R_{i}^{L_{1}}=R_{i}^{\max }$, since $R_{i}^{\max } \geq R_{i}^{L_{2}} \geq R_{i}^{L_{1}}=R_{i}^{\max }$, we have $R_{i}^{L_{2}}=R_{i}^{\max }=R_{i}^{L_{1}}$. Therefore

$$
U_{i}\left[\Gamma\left(t-1, L_{1}\right)\right]=U_{i}\left[\Gamma\left(t, L_{2}\right)\right] .
$$

So, if $\tilde{R}_{i}{ }^{t}>R_{i}^{t}$, we still have $U_{i}\left[\Gamma\left(t-1, L_{1}\right)\right] \geq$ $U_{i}\left[\Gamma\left(t, L_{2}\right)\right]$.

In all, we can show that $U_{i}\left[\Gamma\left(t-1, L_{1}\right)\right] \geq U_{i}\left[\Gamma\left(t, L_{2}\right)\right]$. This completes the proof.

With Lemma 2, we can now show that the best strategy of each user is to report his/her true optimal demand at every clock.

Theorem 2 (Cheat-Proof): Reporting true optimal demand at every clock is a mutually best response for every user, i.e., $U_{i}\left[\Gamma\left(L_{3}, L_{3}\right)\right] \leq U_{i}\left[\Gamma\left(-1, L_{4}\right)\right] \forall i$.

Proof: If all other users report their true optimal demands in every clock, according to Lemma 2 , we have $U_{i}\left[\Gamma\left(L_{3}, L_{3}\right)\right] \leq$ $U_{i}\left[\Gamma\left(L_{3}-1, \tilde{L}_{3}\right)\right] \leq \cdots \leq U_{i}\left[\Gamma\left(-1, L_{4}\right)\right]$, where $\tilde{L}_{3}$ stands for the final clock index of the following scenario: from clock 0 to clock $L_{3}-1, u_{i}$ reports $\tilde{R}_{i}{ }^{0}, \ldots, \tilde{R}_{i}{ }^{L_{3}-1}$, and from clock $L_{3}$ to the final clock $\tilde{L}_{3}, u_{i}$ reports $R_{i}^{L_{3}}, \ldots, R_{i}^{\tilde{L}_{3}}$. Since all users are non-collaborative, reporting true optimal demand at every clock is a mutually best response for every user. There is no incentive for the users to cheat since any cheating may lead to a loss in utility. Therefore, the proposed scheme is cheat-proof. This completes the proof.

In the above theorem, we give a theoretical proof for the cheat-proof strategy. In Section VI, we will verify this cheatproof strategy through experimental results.

\section{EXPERIMENTAL RESULTS}

In order to evaluate the proposed game-theoretic multiuser rate allocation game, we conduct experiments on real video data. Seven video sequences: Akiyo, Mobile, Table, Carphone, Coastguard, Foreman, and Football in QCIF format, are tested. Notice that these video sequences include slow, medium or fast motion, and smooth or complex scene. We use the state-of-art H.264 JM 9.0 video codec to encode the video sequences [24]. By changing the quantization parameter $(\mathrm{QP})$ or using the rate control feature, we are able to compress the video sequences at different bit-rate and achieve different quality requirements.

\section{A. Parameter Estimation}

From Section II, we can see that there are several parameters in our framework, $\gamma_{i}, \beta_{i}, R_{i}^{\min }$, and $R_{i}^{\max }$. In this subsection, we will discuss how to estimate these parameters.

According to (3) and (6), we have

$$
\begin{aligned}
P S N R_{i} & =10\left(\log _{10} e\right) \ln \frac{255^{2}}{D_{i}} \\
& =\left(10 \log _{10} e\right)\left(\gamma_{i}+\beta_{i} R_{i}\right) .
\end{aligned}
$$

Therefore, we can estimate $\gamma_{i}$ and $\beta_{i}$ using offline training. For each video sequence, we first generate a set of $\left(P S N R_{i}, R_{i}\right)$ by encoding the sequence using H.264 JM 9.0 with different QP. Then, the optimal $\gamma_{i}^{\star}$ and $\beta_{i}^{\star}$ can be computed by

$$
\begin{aligned}
& \left(\gamma_{i}^{\star}, \beta_{i}^{\star}\right)= \\
& \quad \min _{\gamma_{i}, \beta_{i}} \sum_{j}\left[P S N R_{i}(j)-\left(10 \log _{10} e\right)\left(\gamma_{i}+\beta_{i} R_{i}(j)\right)\right]^{2}
\end{aligned}
$$

where $j$ is the index of the training set.

Through the training data and equation above, we get the optimal $\gamma_{i}^{\star}$ and $\beta_{i}^{\star}$ for different video sequences and show them in Table I. As shown in Fig. 4, with the optimal $\gamma_{i}^{\star}$ and $\beta_{i}^{\star}$, the $\left(10 \log _{10} e\right)\left(\gamma_{i}+\beta_{i} R_{i}\right)$ can approximate $P S N R_{i}$ well. Due to the page limitation, we only show the results for Football and Coastguard. Similar results are observed for other sequences.

After finding the optimal $\gamma_{i}^{\star}$ and $\beta_{i}^{\star}$, we derive the values for $R_{i}^{\min }$ and $R_{i}^{\max }$. Suppose that the minimal desired PSNR (quality) constraint is $P S N R^{\mathrm{min}}$, e.g., $30 \mathrm{~dB}$, and the maximal satisfied PSNR (quality) constraint is $P S N R^{\max }$, e.g., $45 \mathrm{~dB}$. According to (34), we have

$$
\begin{aligned}
& R_{i}^{\min }=\frac{1}{\beta_{i}^{\star}}\left(\frac{P S N R^{\min }}{10 \log _{10} e}-\gamma_{i}^{\star}\right) \\
& R_{i}^{\max }=\frac{1}{\beta_{i}^{\star}}\left(\frac{P S N R^{\max }}{10 \log _{10} e}-\gamma_{i}^{\star}\right) .
\end{aligned}
$$

According the equations above, the $R_{i}^{\min }$ and $R_{i}^{\max }$ for different sequences are obtained and shown in Table I. From Table I, we can see that the tested video sequences can be classified to four categories according to $\beta^{\star}, R_{i}^{\min }$ and $R_{i}^{\max }$ : slow motion and smooth scene (Akiyo), medium motion and smooth scene (Carphone, Foreman, and Table), medium motion and complex scene (Coastguard), and fast or complex motion (Football and Mobile). 


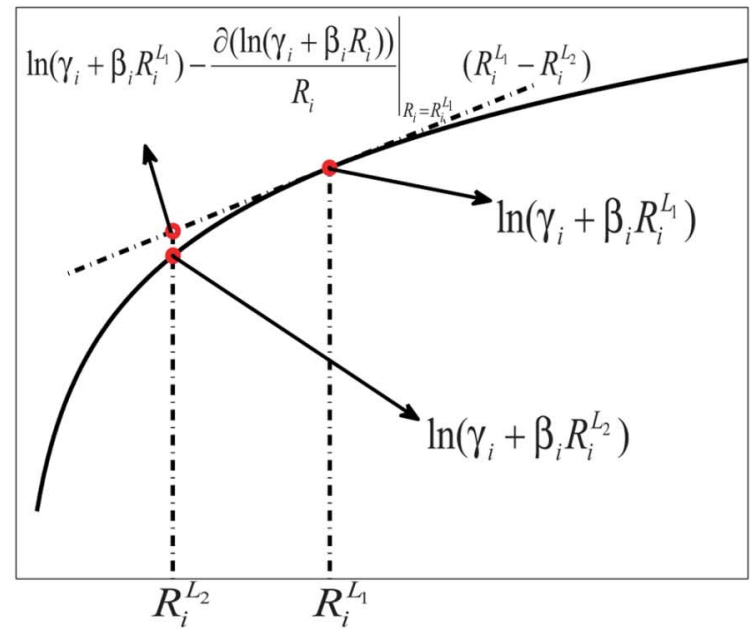

(a)

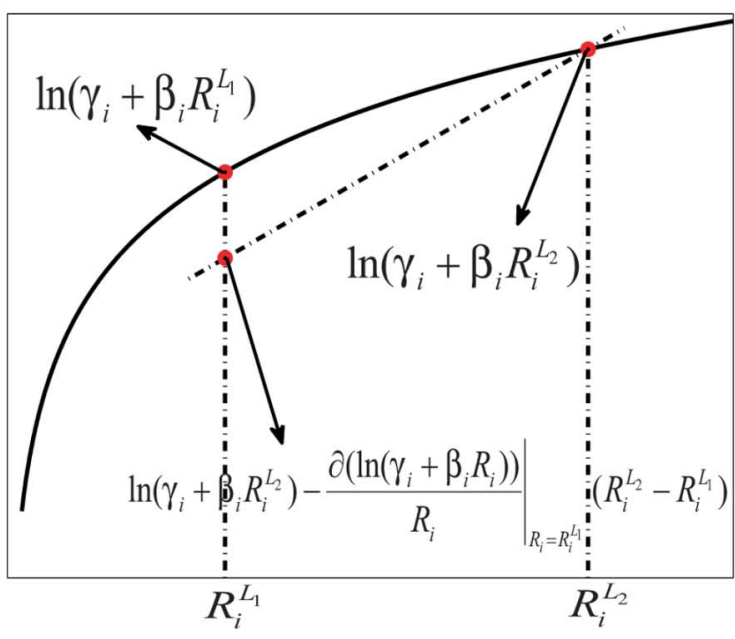

(b)

Fig. 3. Illustration for the proof of Lemma 2: (a) If $\tilde{R}_{i}{ }^{t} \leq R_{i}^{t}$ and $R_{i}^{L_{1}}>\quad R_{i}^{\text {min }}$, we can see that $\left[\ln \left(\gamma_{i}+\beta_{i} R_{i}^{L_{1}}\right)-\left(\partial \ln \left(\gamma_{i}+\beta_{i} R_{i}\right) /\left.\partial R_{i}\right|_{R_{i}=R_{i}^{L_{1}}}\right)\left(R_{i}^{L_{1}}-R_{i}^{L_{2}}\right)\right] \geq \ln \left(\gamma_{i}+\beta_{i} R_{i}^{L_{2}}\right)$. (b) If $\tilde{R}_{i}{ }^{t}>R_{i}^{t}$ and $R_{i}^{L_{1}}<R_{i}^{\text {max }}$, we can see that $\ln \left(\gamma_{i}+\beta_{i} R_{i}^{L_{1}}\right) \geq\left[\ln \left(\gamma_{i}+\beta_{i} R_{i}^{L_{2}}\right)-\left(\partial \ln \left(\gamma_{i}+\beta_{i} R_{i}\right) /\left.\partial R_{i}\right|_{R_{i}=R_{i}^{L}} ^{L_{1}}\right)\left(R_{i}^{L_{2}}-R_{i}^{L_{1}}\right)\right]$.

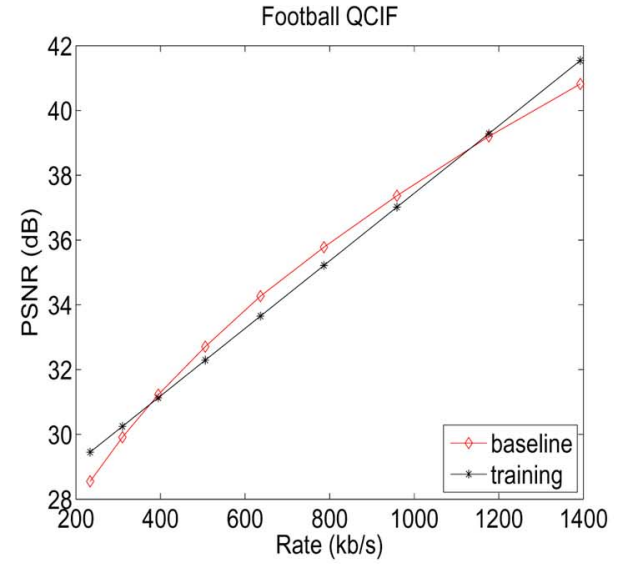

(a)

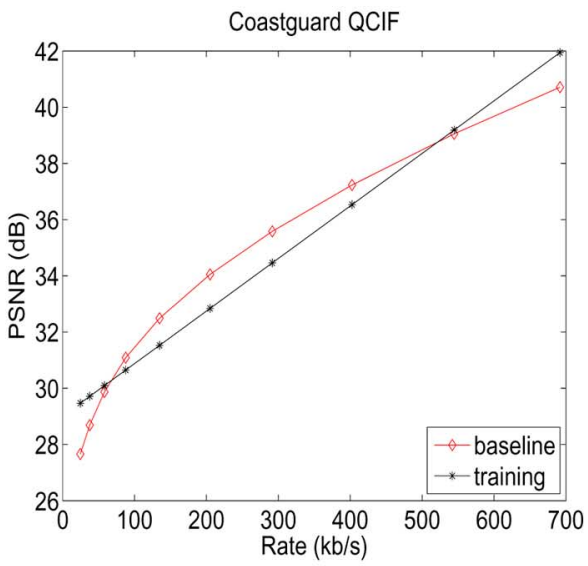

(b)

Fig. 4. Training $\gamma$ and $\beta$. (a) Football. (b) Coastguard.

TABLE I

$\gamma_{i}^{\star}, \beta_{i}^{\star}, R_{i}^{\min }(\mathrm{kb} / \mathrm{s})$, AND $R_{i}^{\max }(\mathrm{kb} / \mathrm{s})$ FOR DIFFERENT SEQUENCE BY TRAINING

\begin{tabular}{|c|c|c|c|c|}
\hline Sequence & $\gamma_{i}^{\star}$ & $\beta_{i}^{\star}$ & $R_{i}^{\text {min }}(\mathrm{kb} / \mathrm{s})$ & $R_{i}^{\text {max }}(\mathrm{kb} / \mathrm{s})$ \\
\hline Akiyo & 6.8449 & 0.0416 & 1.5119 & 84.5447 \\
\hline Carphone & 6.6759 & 0.0114 & 20.2554 & 322.0153 \\
\hline Coastguard & 6.6796 & 0.0043 & 28.4987 & 878.8011 \\
\hline Foreman & 6.7418 & 0.0093 & 17.8168 & 388.7091 \\
\hline Football & 6.2201 & 0.0024 & 286.311 & 1720 \\
\hline Mobile & 6.3464 & 0.0025 & 225.0682 & 1610 \\
\hline Table & 6.8135 & 0.0074 & 12.7781 & 481.1014 \\
\hline
\end{tabular}

\section{B. Multiuser Rate Allocation}

We compare the proposed method with three approaches: the absolute fairness in rate (AFR), which equally divides the available bandwidth to all the users, the absolute fairness in distortion (AFD), which minimizes the maximal distortion of all the users, i.e., min-max fairness, and the approach maximizing the sum of the PSNRs (MSPSNR), i.e., the traditional optimization-based approach shown in (2) with uniform weights. Notice that for AFR, AFD, and MSPSNR, the allocated rate should be within $\left[R_{i}^{\min }, R_{i}^{\max }\right]$. Otherwise, we set it to be $R_{i}^{\min }$ or $R_{i}^{\max }$ and re-allocate the rest rate for the other users. Given the video sequences to be transmitted, the available bandwidth $R$, we can compute the rate allocated to each video sequence using different methods, i.e., AFD, AFR, MSPSNR, and the proposed method. Then, setting the allocated bit-rate as the target bit-rate, we compress the video sequence using the rate control feature in H.264 JM 9.0 reference software. Finally, each user transmits the compressed bitstream to the corresponding receiver.

In the experiments, we assume that there are seven users $u_{1}, u_{2}, \ldots, u_{7}$. They transmit Akiyo, Carphone, Coastguard, Foreman, Table, Football, and Mobile to seven receivers $r_{1}, r_{2}, \ldots, r_{7}$, respectively. We test $R$ at 1000, 2000, 3000, 4000 , and $5000 \mathrm{~kb} / \mathrm{s}$. The allocated bit-rate for each video sequence in different situations (i.e., different $R$ ) using different methods (i.e., AFD, AFR, MSPSNR, and the proposed method) are shown in Fig. 5. From Fig. 5, we can see that AFR equally allocates the bandwidth to each users if the allocated 

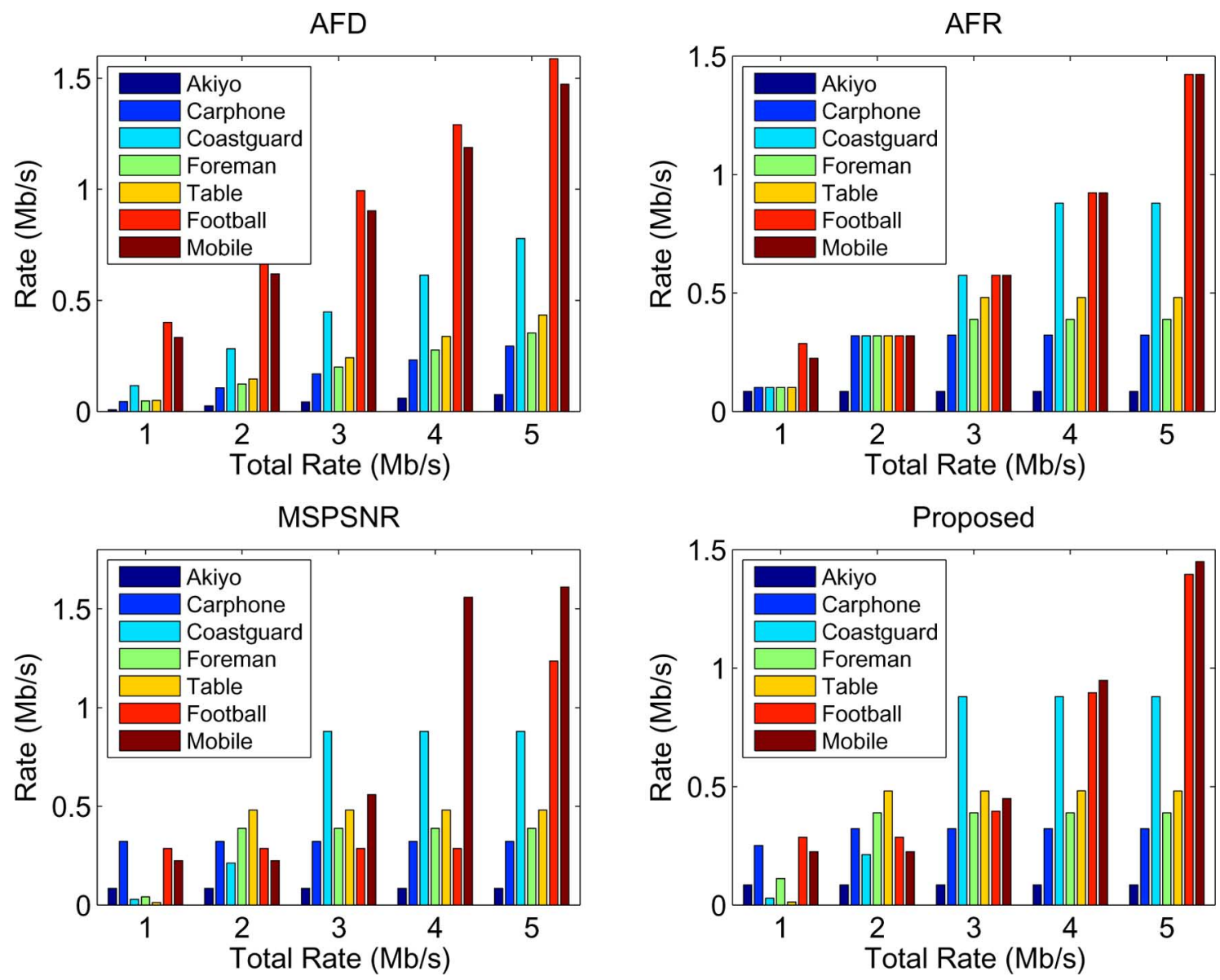

Fig. 5. Allocated rates for Akiyo, Carphone, Coastguard, Foreman, Table, Football, and Mobile using different methods.

bit-rate is within $\left[R_{i}^{\min }, R_{i}^{\max }\right]$. AFD tries to allocate more bit-rate to the video sequence that has more complex motion and/or scene (a smaller $\beta^{\star}$ ) to preserve constant quality among different users. On the contrary, MSPSNR favors the video sequence that has a larger $\beta^{\star}$ since allocating more bit-rate to the sequence with a larger $\beta^{\star}$ leads to a greater increase in the sum of the PSNRs. However, with MSPSNR, the sequence with $\beta_{i}^{\star}$ will not be allocated more bit-rate than $R_{i}^{\min }$ if there is a sequence with $\beta_{j}^{\star}>\beta_{i}^{\star}$ who has not been allocated its maximal rate requirement $R_{j}^{\max }$ yet. Specifically, the rate controller will first allocate each user with $R_{i}^{\min }$. Then, the remaining rates will be first allocated to Akiyo until the bit-rate of Akiyo achieves its maximal requirement. If there are still some unused rates, then Carphone will be satisfied first. The bit-rate of Football with the smallest $\beta^{\star}$ stays at its minimal requirement until all other sequences with higher $\beta^{\star}$ have achieved their maximal rate requirements. Obviously, this is not fair to the users who transmit the sequences with smaller $\beta^{\star}$. By taking the proportional fairness into account, the proposed method can avoid this disadvantage and balance the rate allocation between the sequences with a larger $\beta^{\star}$ and a smaller $\beta^{\star}$. For example, as shown in Fig. 5, when the total available network bandwidth $R$ increases from $3000 \mathrm{~kb} / \mathrm{s}$ to $4000 \mathrm{~kb} / \mathrm{s}$, both the bit-rate of Mobile and Football increase. This is because the proposed method with the proportional fairness criterion aims at maximizing the product of the utility function $U_{i}$, and

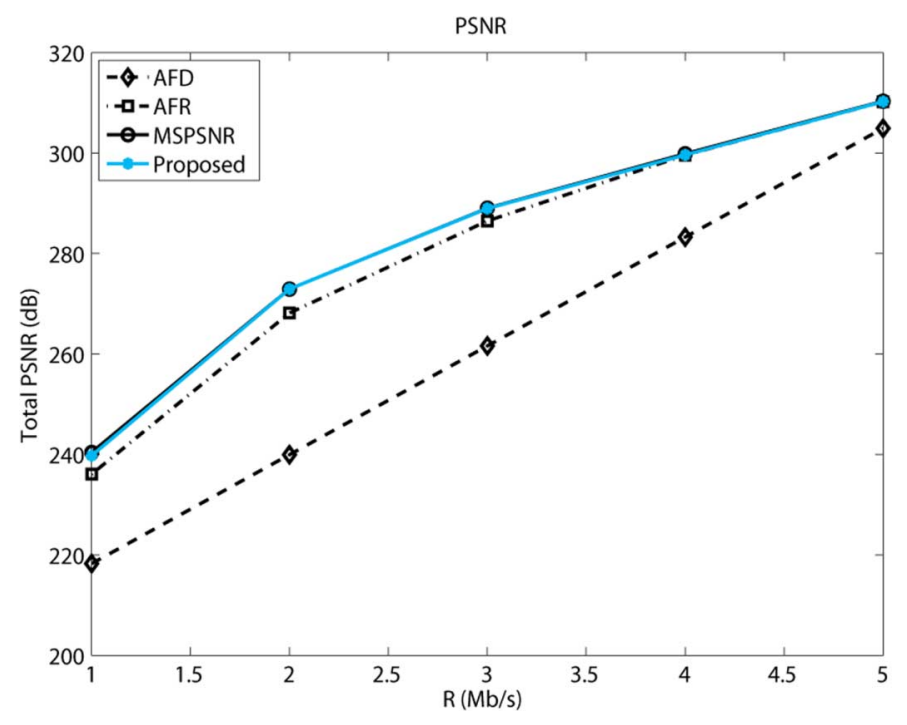

Fig. 6. Sum of PSNR versus the available network bandwidth $R$.

keeping a certain balance between the sequences with a larger $\beta^{\star}$ and a smaller $\beta^{\star}$ leads to an increase in the product.

Let $T_{-} P S N R=\sum_{i=1}^{N} P S N R_{i}$ be the sum of the analytical $P S N R_{i}$ computed by (34) of all the users. In Fig. 6, we show $T_{-} P S N R$ versus the available network bandwidth $R$. We can see that there is a big gap between the performance of AFD, 


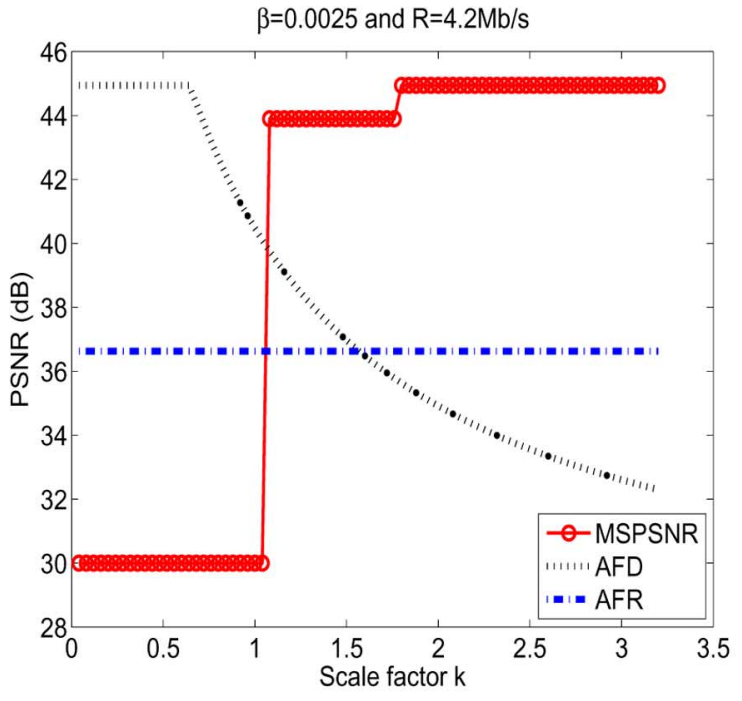

(a)

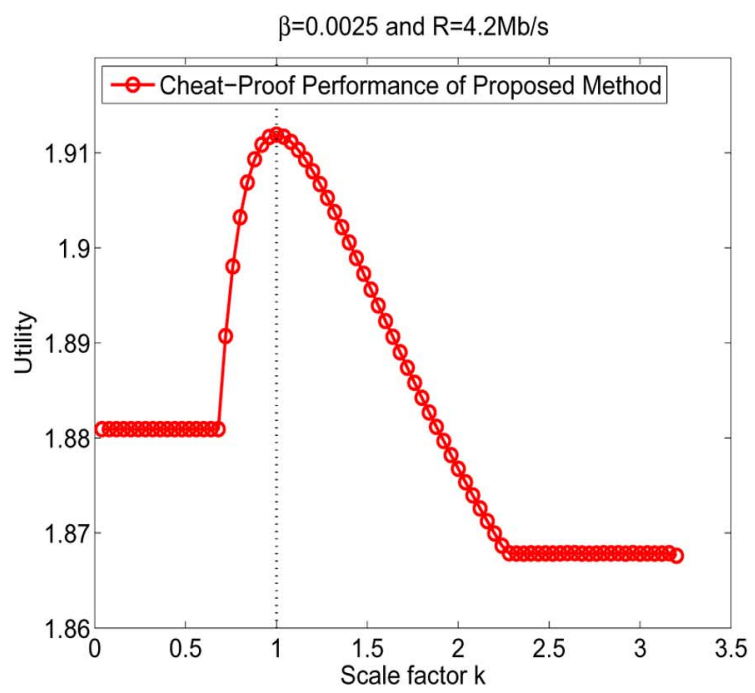

(b)

Fig. 7. Cheat-proof performance. (a) AFD, AFR, and MSPSNR. (b) Proposed method.

AFR, and MSPSNR, which means using AFD or AFR leads to a big loss in the system performance. However, the performance of the proposed method is almost the same as that of MSPSNR, which fully demonstrates the efficiency of the proposed method. Therefore, while achieving a fair rate allocation among different users, the proposed method still performs well in terms of total PSNR.

Finally, we evaluate the cheat-proof property of different methods. As shown in Table I, since $\beta$ is the most important parameter representing the characteristics of video sequences, the best way for $u_{i}$ to pretend as another user $u_{j}$ is to use $\beta_{j}$ rather than $\beta_{i}$ in calculating optimal demand. Therefore, we evaluate the cheat-proof property in terms of $\beta$. In this experiment, the available network bandwidth $R$ is set to be $4.2 \mathrm{Mb} / \mathrm{s}$. We assume that $u_{6}$ who transmits Mobile sequence will cheat while other users are honest. In AFD, AFR, and MSPSNR, $u_{6}$ reports a false $\tilde{\beta}$ to the controller by scaling the original $\beta$ with a factor $k$, i.e., $\tilde{\beta}=k \beta$. In the proposed method, at each clock $t$ of the auction, $u_{6}$ uses $\tilde{\beta}$ to generate the "optimal" demand $\tilde{R}_{6}{ }^{t}$ using $\tilde{R}_{6}{ }^{t}=\max \left[R_{6}^{\min }, \min \left(\left(1 / a^{t}\right)-\left(\gamma_{6} / k \beta_{6}\right), R_{6}^{\max }\right)\right]$ and reports ${\tilde{R_{6}}}^{t}$ to the controller. As shown in Fig. 7(a), the PSNR performance of AFR is independent of the scale factor $k$. This is because AFR does not care about $\beta$ and just equally allocates the bandwidth to each user if the allocated bit-rate is within $\left[R_{i}^{\min }, R_{i}^{\max }\right]$. The PSNR performance of AFD decreases as $k$ increases. This is because AFD tries to allocate more bit-rate to the video sequence with a smaller $\beta$ to preserve constant quality among different users. Therefore, with AFD, all users tend to report a smaller $\beta$ to the controller to obtain a better PSNR performance. On the contrary, the PSNR performance of MSPSNR is an increasing piecewise constant function in terms of $k$. This is because, with MSPSNR, the sequence with $\beta_{i}$ will not be allocated more bit-rate than $R_{i}^{\min }$ if there is a sequence with $\beta_{j}>\beta_{i}$ who has not been allocated its maximal rate requirement $R_{j}^{\max }$ yet. To be allocated more rate and obtain a higher PSNR, $u_{6}$ should increase $k$ until at least $k \beta_{6}>\beta_{j}$ where $\beta_{j}=\min _{l}\left(\beta_{l}>\beta_{6}\right)$. Therefore, with MSPSNR, all users tend to report a larger $\beta$ to the controller to obtain a better PSNR performance. However, with the proposed method, as shown in Fig. 7(b), reporting the optimal demand generated by the true $\beta(k=1)$ will lead to the best utility. Any deviation will lead to a loss in terms of utility, which means that the proposed method is cheat-proof. Therefore, the proposed method ensures all users will be honest about their private information.

\section{CONCLUSIONS}

In this paper, we proposed a game-theoretic framework for multiuser multimedia rate allocation and a distributed cheatproof scheme for the users to converge to the NE of the game. Different from the traditional optimization-based approaches, which mainly focused on the efficiency issue, e.g., maximizing the system performance, the proposed method not only considered the efficiency issue but also the fairness issue. From the experimental results on the real video sequences, we could see that with the proportional fairness criterion, the proposed game-theoretic method could efficiently and fairly allocate bit-rate to different users by allocating more bit-rate to the sequence with slower motion and/or simpler scene while keeping an eye on the fast motion and/or complex scene sequence. We also found that, with the proposed distributed cheat-proof rate allocation scheme, reporting the true optimal demand at every clock is the mutual best response for every user. Moreover, we showed that the traditional optimization-based method that maximizes the weighted sum of the PSNRs is a special case of the game-theoretic framework with the utility function defined as an exponential function of PSNR.

\section{REFERENCES}

[1] J. R. Corbera and S. Lei, "Rate control in DCT video coding for lowdelay communications," IEEE Trans. Circuits Syst. Video Technol., vol. 9, no. 1, pp. 172-185, Feb. 1999. 
[2] T. H. Chiang and Y. Q. Zhang, "A new rate control scheme using quadratic rate distortion model," IEEE Trans. Circuits Syst. Video Technol., vol. 7, no. 1, pp. 246-250, Feb. 1997.

[3] W. Ding and B. Liu, "Rate control of MPEG video coding and recording by rate-quantization modeling," IEEE Trans. Circuits Syst. Video Technol., vol. 6, no. 1, pp. 12-20, Feb. 1996.

[4] T. V. Lakshman, A. Ortega, and A. R. Reibman, "VBR video: Tradeoffs and potentials," Proc. IEEE, vol. 86, no. 5, pp. 952-973, May 1998.

[5] Z. He and S. K. Mitra, "A unified rate-distortion analysis framework for transform coding," IEEE Trans. Circuits Syst. Video Technol., vol. 11, no. 12, pp. 1221-1236, Dec. 2001.

[6] X. J. Zhao, Y. W. He, S. Q. Yang, and Y. Z. Zhong, "Rate allocation of equal image quality for MPEG-4 FGS video streaming," in Proc. Packet Video (PV), 2002.

[7] X. Zhang, A. Vetro, Y. Q. Shi, and H. Sun, "Constant quality constrained rate allocation for FGS video coded bitstreams," in Proc. SPIE Conf. Visual Communications and Image Processing (VCIP), 2002.

[8] A. Ortega and K. Ramchandran, "Rate-distortion techniques in image and video compression," IEEE Signal Process. Mag., vol. 15, no. 6, pp. 25-50, Nov. 1998.

[9] C. Shen and M. van der Schaar, "Optimal resource allocation for multimedia applications over multiaccess fading channels," IEEE Trans. Wireless Commun., vol. 7, no. 9, pp. 3546-3557, Sep. 2008.

[10] W. Rhee and J. M. Cioffi, "Increase in capacity of multiuser OFDM system using dynamic subchannel allocation," in Proc. IEEE Veh. Technol. Conf., 2000.

[11] Z. Han, Z. Ji, and K. J. R. Liu, "Fair multiuser channel allocation for OFDMA networks using Nash bargaining solutions and coalitions," IEEE Trans. Commun., vol. 53, no. 8, pp. 1366-1376, Aug. 2005.

[12] H. Park and M. van der Schaar, "Bargaining strategies for networked multimedia resource management," IEEE Trans. Signal Process., vol. 55, no. 7, pp. 3496-3511, Jul. 2007.

[13] C. U. Saraydar, N. B. Mandayam, and D. J. Goodman, "Efficient power control via pricing in wireless data networks," IEEE Trans. Commun., vol. 50, no. 2, pp. 291-303, Feb. 2002.

[14] L. M. Ausubel, "An efficient ascending-bid auction for multiple objects," Amer. Econ. Rev., vol. 94, pp. 1452-1475, 2004.

[15] H. M. Hang and J. J. Chen, "Source model for transform video coder and its application-Part i: Fundamental theory," IEEE Trans. Circuits Syst. Video Technol., vol. 7, no. 2, pp. 287-298, Apr. 1997.

[16] M. Dai, D. Loguinov, and H. Radha, "Rate-distortion modeling of scalable video coders," in Proc. IEEE Int. Conf. Image Processing, 2004.

[17] K. Stuhlmuller, N. Farber, M. Link, and B. Girod, "Analysis of video transmission over lossy channels," IEEE J. Select. Areas Commun., vol. 18, no. 6, pp. 1012-1032, Jun. 2000.

[18] Y. Chen and O. C. Au, "Simultaneous RD-optimized rate control and video de-noising," in Proc. IEEE Int. Conf. Acoustics, Speech and Signal Processing, 2008.

[19] Z. G. Li, F. Pan, K. P. Lim, X. Lin, and S. Rahardja, "Adaptive rate control for H.264," in Proc. IEEE Int. Conf. Image Processing, 2004.

[20] M. Wang and M. van der Schaar, "Rate-distortion modeling for wavelet video coders," in Proc. IEEE Int. Conf. Acoustics, Speech and Signal Processing, 2005.

[21] F. Kelly, "Charging and rate control for elastic traffic," Eur. Trans. Telecommun., vol. 28, pp. 33-37, 1997.

[22] S. Boyd and L. Vandenberghe, Convex Optimization. Cambridge, U.K.: Cambridge Univ. Press, 2004.

[23] Z. Han, Z. Ji, and K. J. R. Liu, "A cartel maintenance framework to enforce cooperation in wireless networks with selfish users," IEEE Trans. Wirless Commun., vol. 7, no. 5, pp. 1889-1899, May 2008.

[24] H.264/AVC Software. [Online]. Available: http://iphome.hhi.de/ suehring/tml/.

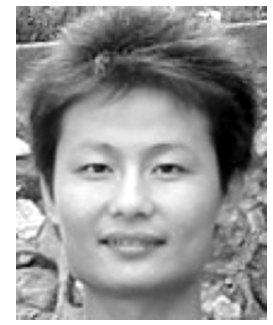

Yan Chen (S'06) received the Bachelor's degree from University of Science and Technology of China (USTC), Hefei, in 2004 and the M.Phil. degree from Hong Kong University of Science and Technology (HKUST), Clear Water Bay, Hong Kong, in 2007. Now he is pursuing the Ph.D. degree in the Department of Electrical and Computer Engineering at the University of Maryland, College Park.

$\mathrm{He}$ interned in the Internet Media group of Microsoft Research Asia (MSRA) from July to October 2004. His current research interests are in multimedia signal processing, cooperative multimedia communication and networking, multimedia social network, and multimedia forensic.

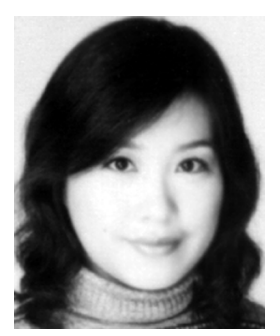

Beibei Wang (S'07) received the B.S. degree in electrical engineering (with highest honors) from the University of Science and Technology of China, Hefei, in 2004, and the Ph.D. degree in electrical engineering from the University of Maryland, College Park, in 2009.

She is currently a research associate at the University of Maryland. Her research interests include dynamic spectrum allocation and management in cognitive radio systems, wireless communications and networking, game theory, wireless multimedia, and net-

work security.

Dr. Wang was the recipient of the Graduate School Fellowship, the Future Faculty Fellowship, and the Dean's Doctoral Research Award from the University of Maryland.

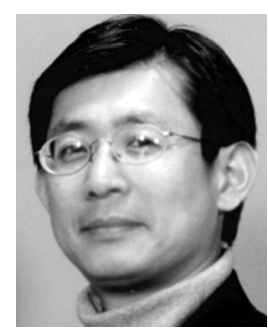

K. J. Ray Liu (F'03) is a Distinguished Scholar-Teacher of the University of Maryland, College Park. He is Associate Chair of Graduate Studies and Research of the Electrical and Computer Engineering Department and leads the Maryland Signals and Information Group conducting research encompassing broad aspects of information technology including communications and networking, information forensics and security, multimedia signal processing, and biomedical technology. His recent books include Cooperative Communications and Networking (Cambridge, U.K.: Cambridge Univ. Press, 2008); Resource Allocation for Wireless Networks: Basics, Techniques, and Applications (Cambridge, U.K.: Cambridge Univ. Press, 2008); Ultra-Wideband Communication Systems: The Multiband OFDM Approach (Piscataway, NJ: IEEE-Wiley, 2007); Network-Aware Security for Group Communications (New York: Springer, 2007); Multimedia Fingerprinting Forensics for Traitor Tracing (New York: Hindawi, 2005); and Handbook on Array Processing and Sensor Networks (Piscataway, NJ: IEEE-Wiley, 2009).

Dr. Liu is the recipient of numerous honors and awards including best paper awards from the IEEE Signal Processing Society, the IEEE Vehicular Technology Society, and EURASIP; IEEE Signal Processing Society Distinguished Lecturer, EURASIP Meritorious Service Award, and National Science Foundation Young Investigator Award. He also received various teaching and research recognitions from the University of Maryland including university-level Invention of the Year Award; and Poole and Kent Senior Faculty Teaching Award and Outstanding Faculty Research Award, both from A. James Clark School of Engineering Faculty. He is a Fellow of AAAS. He was Vice President-Publications and will serve as President-Elect starting in 2010. He was the Editor-in-Chief of the IEEE Signal PROCESSING MagaZINE and the founding Editor-in-Chief of EURASIP Journal on Applied Signal Processing. 